\title{
Writing an Academic Paper for the Purpose of Publication
}

\author{
Paromita Mazumdar ${ }^{1}$ \\ ${ }^{1}$ Department of Conservative Dentistry and Endodontics, Guru Nanak \\ Institute of Dental Sciences and Research, Kolkata, West Bengal, India.
}

\section{ABSTRACT}

Writing a scientific article is a daunting task but needs to be accomplished to be considered for publication in appropriate journals. A scientific experiment or project is not considered complete unless the outcome is disseminated to the fraternity. The entire work conducted by the researcher has to be written as a scientific document keeping in mind the heterogeneity of readers and the range of journals which consider submission of manuscripts for the purpose of publication. The purpose of publication is not only for getting promotions, evidence build up and peer recognition but also as a measure of work done by competent people in relevant field. The process of article writing should begin from the conceptualisation of the scientific experiment. Penning down a manuscript does not follow the same order in which an article is read. Scientific articles follow the Introduction, Methods, Results and Discussion (IMRaD) format when printed and read, whereas while preparing the manuscript the order is different. The methods are written first since it is the actual work done by a researcher and should be most easy to write. The findings / observations can be written next for the results section. The discussion section interprets the results, puts forward the work of similar research and ends with the conclusions and limitations. The introduction, abstract and title are the last to be written since by then most of the manuscript is well articulated. Selection of journals, acknowledgement, conflict of interest, plagiarism, referencing style and letter to editors are the other aspects of preparation of manuscript.

\section{KEY WORDS}

Publication, Article Writing
Corresponding Author:

Dr. Paromita Mazumdar, HIG- II A, 7/14, 1050/2,

Survey Par, Calcutta Greens Phase-II,

Housing Complex, Kolkata - 700075,

West Bengal, India.

E-mail:pm.evershine@gmail.com

DOI: $10.14260 /$ jemds/2021/319

How to Cite This Article:

Mazumdar P. Writing an academic paper for the purpose of publication. J Evolution Med Dent Sci 2021;10(20):1525-1531, DOI: $10.14260 /$ jemds/2021/319

Submission 09-10-2020,

Peer Review 11-03-2021,

Acceptance 19-03-2021,

Published 17-05-2021.

Copyright (C) 2021 Paromita Mazumdar This is an open access article distributed under Creative Commons Attribution License [Attribution 4.0 International (CC $B Y 4.0)]$ 


\section{BACKGROUND}

Research work is undertaken by students, scholars and faculty members at postgraduate and higher levels. Faculty members are also researchers. Conducting a scientific experiment is as important as documenting the same in an organized manner and eventually write a scientific article in order to be published and make known to peers. Peer validation is the first step of building up evidence on the particular field of work. Robert Day (1983) defines a scientific paper as a written and published report describing original research results. The dissemination of research results and findings is an integral part of the research process. Booth et al. (1995) states three obvious reasons - to remember, because once something is forgotten, it cannot be reproduced correctly without having written notice; to understand, as writing about a subject can only be accomplished by approaching the subject in a structured way, which itself leads to better understanding there of; and to gain perspective, as writing includes looking at something from different points of view. ${ }^{1}$

Writing a scientific article may be intimidating to beginners and experienced alike since there are various aspects to be considered namely, designing the experiment and prepare an outline of work, meticulous record keeping during experiment, searching relevant literature, maintaining timeline, subjecting the results to appropriate statistical analyses and finally critically analyse the findings. It is essential to prepare a good manuscript and select a journal where the manuscript has a chance of acceptance depending on the scope of the journal, originality of the idea, quality of evidence and the importance of findings. This choice it at times influenced by factors like matching the journal's readership, indexing, impact factor, frequency of publication, acceptance rate and publication expenses involved. ${ }^{2}$ The 'instructions to authors section usually provides guidelines that are specific to a particular journal and usually differs from one journal to another. The authors should shortlist few journals during the research work and prepare a framework of the manuscript adhering to the general guidelines of a journal in which the article is intended to be sent. ${ }^{2}$

Sometimes it becomes difficult to maintain a structured sequence of work and preparation of the manuscript in situations where submission of an abstract is required ahead of completion of experiment in case the findings are to be read at a conference and are later prepared for the purpose of publication. In such situations, the title and abstract need to be written for framing the outline of research work (ORW). ${ }^{3}$

Sometimes a synopsis of dissertation is required to be submitted where a title, literature review and brief methodology are written based on a pilot study. Thesis is a detailed description of an original experimental study or project undertaken for a specific academic purpose like prerequisite for being awarded higher degrees. If the write up is intended for synopsis of dissertation or thesis, the format is dictated by the concerning institution, university or organization. 4

The usual contents of a scientific article are: title, abstract, introduction, materials and method, observations and results, statistical analysis, discussions, conclusions, future directions and references (in Vancouver style). Figures, tables and photographs are usually requested to be sent separately with numbering and legends indicating where they are supposed to be inserted in the main text. In addition to the main content a summary, conflict of interest declaration, ethics clearance certificate, author contributions, letter to editor, layman format, what's new etc may be required which varies from publisher to publisher. ${ }^{5}$

This article aims at giving the readers a guideline for preparing a manuscript for intended consideration for publication in a journal.

\section{HOW TO BEGIN}

It is a good idea to divide responsibilities (if there are multiple researchers) pertaining to the study intended for publication at the time of conceptualisation. ${ }^{6}$

\section{Role Divisions May Be as Follows}

- Conceptualisation

- Data curation

- Formal analysis

- Investigation

- Methodology

- Project administration

- Resources

- Software

- Supervision

- Validation

- Visualization

- Writing- original draft

- Writing - review and editing or as

\section{Author Contributions}

a. Conceived the study

b. Wrote the manuscript

c. Coordinated patient recruitment, clinical assessments and collection and follow up data

d. Guided and coordinated parallel sequencing and other experiments where applicable

e. Provided overall supervision including statistical guidance

f. Carried out statistical and bioinformatics of samples.

\section{GETTING STARTED}

\section{Manuscript Components for Publishing}

The typical representation of basic components of a scientific paper writing has been described in a Fish Bone Model by Meo.2 


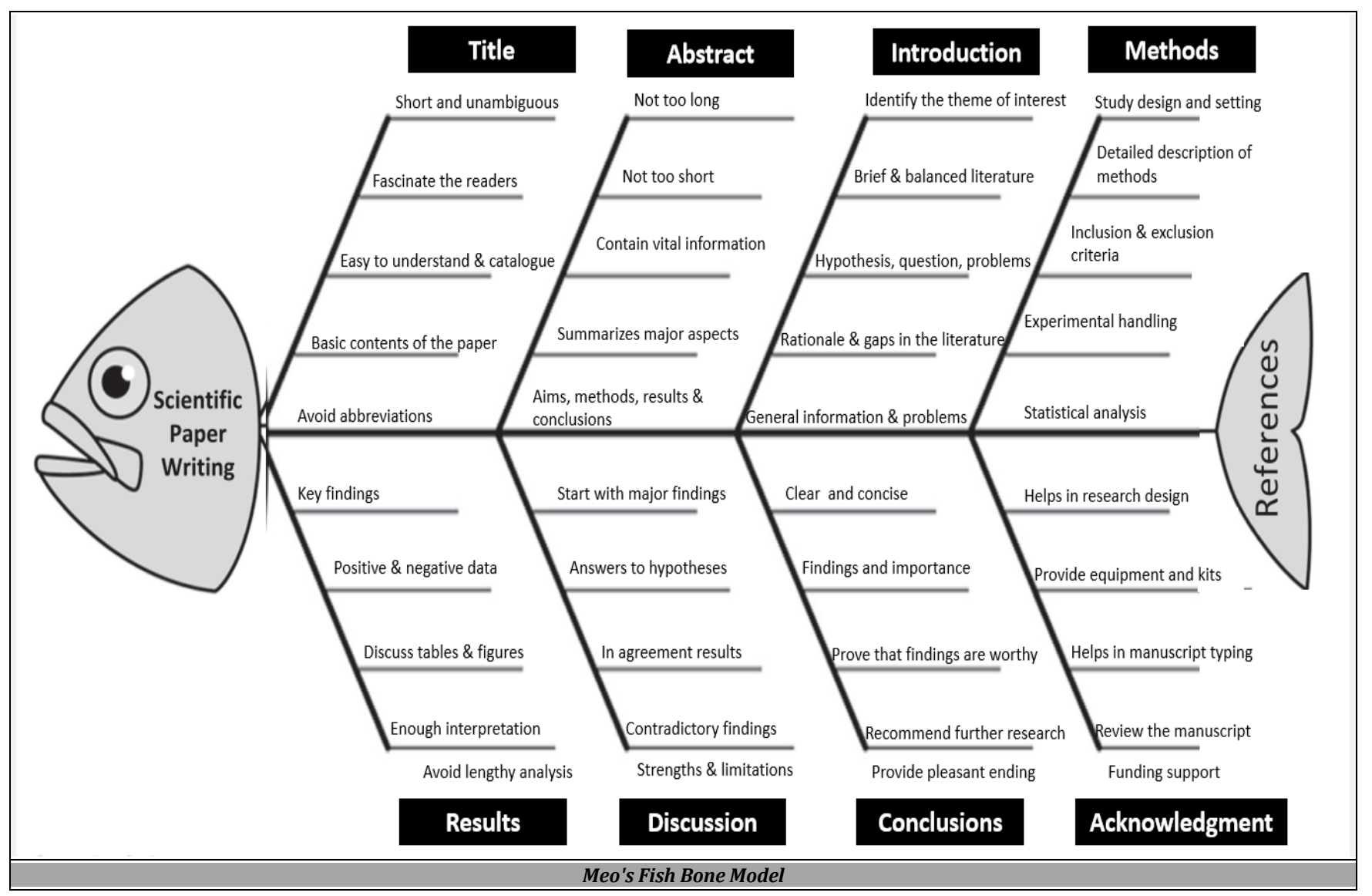

The usual steps of organizing a manuscript are as follows. ${ }^{7}$

1. Preparing a suitable working title and frame a hypothesis

2. Preparing the figures and tables

3. Writing the methods.

4. Writing the results.

5. Writing the discussion

6. Finalizing the results and discussion before writing the introduction should be done because, if the discussion is insufficient, objective demonstration of the scientific significance of work in the introduction cannot be written properly.

7. Writing a clear conclusion.

8. Writing an introduction.

9. Writing the abstract.

10. Selection of keywords for indexing.

11. Writing the acknowledgement.

12. Writing the references.

The author should begin writing the material and method while performing experiments. Two separate note books may be used. One for entering record or observations of the experiment on a daily basis and another for writing review of literature both in the form of exact words and paraphrasing the same.

The rough copy write up should start with general information that applies to entire manuscript and then move on to specific experimental details. The flow and continuity of order in which experiment is conducted should be described. Citations for procedures that have been done by other researchers should be added where applicable. Controls and statistical tests should be described as fully as possible. Thorough, listing equipment/material including their sourcing should be done. Every detail is mentioned so that the experiments can be reproduced without an excess information not affecting the interpretation of the results. All the appropriate controls should be mentioned. To write a paper as a scientific publication is conventionally and internationally named - requires compliance with specific rules, which make it suitable to diffusion and well used by the readers. ${ }^{2}$

Photo documentation should be done at this stage and each experimental procedure picture should be captured. It is also advisable to consult a statistician during the designing of the experiment.

\section{CORE PROCESS OF WRITING}

The title of the scientific paper is the most read and important component of an article which creates the first impression of the article that an editor, reviewer and reader reads to feel interested about the contents of the scientific paper. Title should be short, easy to understand and catalogue with an intent to fascinate the readers. Title must contain the primary key words describing the work presented and reflect the core contents of the manuscript. The title summarises the main idea or ideas of a study and may be attempted limiting it to 14 - 16 words. A good title is succinct and adequately describes the contents and / or purpose of research paper. It is the one that makes first impression with the editor, reviewer and reader. The purpose of the title is that it is the determinant for indexing, visibility of paper and hence citation and should be indicative rather than descriptive in nature. It reflects the research question, essence, novelty of the work accurately captures what was done. 
Hence, it is best created after the research work is completed. However, a rough draft can be prepared ahead so that it is easy to refer to the title and finalise the aim and objectives as the experiment progresses. In situations like conference presentations, synopsis submission, clinical trials or in studies where ethics approval is required before embarking on the study, title should be thoughtfully framed since the title once registered after ethics committee approval cannot be changed. If a change in title is desired, the same has to be ratified by concerning ethics committee. Titles should almost never contain abbreviations, chemical formulas, proprietary (rather than generic) names, jargon, and the like. ${ }^{8}$

\section{FINAL WRITING OF MATERIALS AND METHODS 9}

The methods section is the hero and most important part in the scientific paper. It begins by explaining the overall strategy.

Method should contain detailed information to allow the reader to understand the steps that were done, where was it done, and how was it done. The information available in the methods section of a scientific paper are about the study design, settings, materials and equipment preferably in a tabular form, biological features of control, treatment groups and variables measured. If the study is based on the human model, the age, height, weight, gender, ethnicity, educational and socioeconomic status should be discussed. Information about study protocol, tabulated inclusion and exclusion criteria, sample size and grouping, data collection and replication, pre-experiment, experimental handling, measurements, and procedure detail, and how the findings were summarized in means, percent, SD, SEM, $95 \%$, etc. should be provided. It is necessary to discuss the statistical software used, data computed, analysed and probability developed as a separate statistical analysis section as fully as possible. The author should begin writing the material and method while performing experiments. Citations for procedures that have been described previously should be clearly mentioned. Discussing the pros and cons of methods or results is best avoided. Concise but thorough listing equipment, material, method of use of materials, source of materials and laboratory used should be done. Enough details including controls should be mentioned so that the experiments can be reproduced.

All appropriate citations are included and the source of each material should be listed. After reading methods section, another researcher should be able to duplicate the study. Structured methods sections (i.e., with subheadings such as subjects, treatment protocol, and statistical methods) are popular, and some prestigious journals, which have limited print space, provide an expanded, online methods section. ${ }^{3}$

The photos and other supplementaryterial need to be arranged and legends framed at this point depending on journal instructions.

Reporting guidelines and checklists have been developed for a wide variety of research types and study designs. A guideline is a checklist, diagram or explicit text which guides authors in reporting research, and should be developed using explicit methodology. Reporting guidelines have been developed to help improve the reporting of specific study designs. If followed by authors, this should enable users to understand the design, conduct and analysis of the research, to critically appraise and review the findings and interpret the conclusions appropriately. Some of the reporting guidelines for common study designs are-10

Randomised trials- Consort (Consolidated Standards of Reporting Trials 2010) guideline led by a group of clinical trial methodologists, guideline developers, journal editors and knowledge translation specialist is intended to enable readers to understand a trial's design, conduct, analysis and interpretation, and to assess the validity of its results. This can only be achieved through complete adherence and transparency by authors.

Observational studies- The Strengthening the Reporting of observational studies in Epidemiology (STROBE)

Systematic reviews- Preferred Reporting Items for Systematic Reviews and Meta-Analyses (PRISMA)

\section{Study Protocols - Standard Protocol Items - Recommendations for International Trials (SPIRIT) ${ }^{10-11}$}

- $\quad$ Case reports- CARE (Care Report)

- Clinical practice guidelines- The appraisal of guidelines for research and evaluation (AGREE)

- Qualitative research- Standards for reporting qualitative research (SRQR)

- Animal pre-clinical studies-Animal research reporting of in vivo experiments (ARRIVE)

- Quality improvement studies-Standards for quality improvement reporting excellence (SQUIRE)

- Economic evaluations-Consolidated health economic evaluation reporting standards (CHEERS)

- Preferred reporting items for study designs in endodontology (PRIDE) is a new online web-based resource that has been developed to increase awareness of importance of high standards when writing research and clinical reports in Endodontology.

- Editors and peer reviewers may use these checklists when assessing manuscript better as they will more easily understand what has been done.

\section{OBSERVATION TABLES AND FIGURES}

Tables and figures are an integral part of a well-documented and well written scientific paper. The bulk of the detailed information in a paper is usually presented in its tables. Tables provide exact values with a possibility to present complex data and analyses in a familiar format, figures provide a quick visual impression, useful to illustrate complex relationships and general comparisons but with less precision. Infographics are a nice and attractive method of representing data but it should be borne in mind that the representation should be applicable to the provided data set. For example, bar graphs are most appropriate for numerical data whereas pie charts are suitable for representing percentages. Most of the editors and reviewers have a habit of first glancing through info graphics. 
No information should be repeated and the illustrations should not duplicate the information described elsewhere in the manuscript. 12

\section{RESULTS}

Results need to be categorised and classified and made to interact with each other and subjected to appropriate statistical tools to arrive at an objective conclusion. ${ }^{13}$

\section{DISCUSSION}

At the point of writing the discussion, a hypothesis has been examined, data collected and analyzed and results summarized. In this section, implications of the results are evaluated and interpreted, with respect to the original research question. Meaning, purpose, and practical relevance of the findings are disclosed to the reader, attempting to convince the reader of the study's merit. Answer to why for every observation makes up the discussion. ${ }^{6}$

It is best to not try to establish facts from data analysis and trends in isolation without the aid of references. Best discussion is analytical and not descriptive, built on references coherently to validate the new findings from data analysis of trends. One should start by describing in simple terms what the data shows. The author should refer to statistical analysis such as significance or goodness of fit.

\section{THE PACKAGING}

Introduction (3 paragraphs), abstract, keywords (5 words), running title and cover letter are the marketing tools to place the manuscript in a succinct manner thereby increasing chances of publication and subsequent visibility. ${ }^{14}$

\section{Introduction ${ }^{15}$}

The introduction to a research paper introduces the topic being researched. The introduction contains a topic sentence, a thesis statement, three to five reasons, details and/or facts supporting the research followed by a conclusion. It should be brief, concise and clear in approximately three paragraphs. The various components of an introduction include;

1. Significance of the problem

2. Status of the issue

3. Analysis of the report

4. Gap or conflict in current knowledge

5. Problem statement

6. Hypothesis

7. Aim, objective and scope

A research hypothesis is a specific, clear, and testable proposition or predictive statement about the possible outcome of a scientific research study based on a particular property of a population or experimental groups, such as presumed differences between groups on a variable or relationships between variables. The hypothesis is a prediction which is more than a guess. Most of the time, the hypothesis begins with a question which is then explored through background research. It is only at this point that researchers begin to develop a testable and verifiable hypothesis. Unless an exploratory study is created, hypothesis should always explain what is expected to happen.

Objective and scope are often confused. Research objectives outline the specific steps one will take to achieve research aim. Objectives define the what, why, who, when and how questions. Objective is a single point agenda and is specific, concrete, and quantifiable in nature. Sometimes aim and objective are used interchangeably. Scope is concerning the specifics, boundaries or to what scale the given objective is to be realised. The last paragraph of the introduction may be written as 'in the present investigation an attempt has been made to...........'.

\begin{abstract}
For an original research, abstract is written within word limit provided by publisher under sub headings of introduction, materials methods, results and conclusion. For a review article, it may a single paragraph. As publication manual of the American Psychological Association states (2012: 12), an abstract is a brief, comprehensive summary of the content of the article; it allows reader to survey the tents of the article quickly and like a title, it enables abstracting and information services to index and retrieve articles. It is outline of the research work and not the conclusion, usually 200-300 words. It sets tone for the entire paper. The purpose is data base searching of the article, high visibility, consistent, accurate, quick indexing and retrieval of digital asset content. Resourceful in articles and abstract, controlled to vocabulary, thesaurus used for indexing articles in Pub Med, Medical Subject Heading (MeSH) in the National library of Medicine (NLM). Cataloguers, taggers, indexers use these keywords. ${ }^{16,17}$
\end{abstract}

\section{Final Structure ${ }^{1}$}

The 'Hourglass Model' (light-gray parts) and the 'King Model' represents an extended set of parts in a typical paper's structure. ${ }^{1}$

\section{THE FINALE}

\section{Cover Letter to the Editor. ${ }^{15}$}

It is marketing of research ideas and needs to have the following elements

1. Defined objective of the work.

2. Relevance of the work related to the objectives and scopes of the journal.

3. Relevant earlier publications in the journal.

4. Justification of how the manuscript fills the knowledge gap or will disseminate fresh concept.

5. Validated checklists are important instruments for the screening process based on different criteria (linked to the publisher organization). The checklist includes research concept and design, collection, data assembly, data analysis and interpretation, writing the article, critical revision of article and finalization and upload. Submissions may also contain supplemental material (if any like images or tables or charts) that needs to be 
prepared and uploaded separately from the main manuscript content as per the journal guidelines.

\section{HEART BREAK}

\section{Reasons for Rejection.18}

- Inappropriate interpretation of findings

- Research design

- Defects in methodology (even intricate details are important)
- Results are unoriginal, trivial or predictable

- Inappropriate or inadequate presentation of data

- Confused rationale (both support and negation need to be clear)

- Poor or inadequate sample size or selection

- Failure to collect data in variables that influence results

- Discussion missing a critical analysis component

- Lack of innovation or novelty

- Writing style, grammar, format, sentence structure

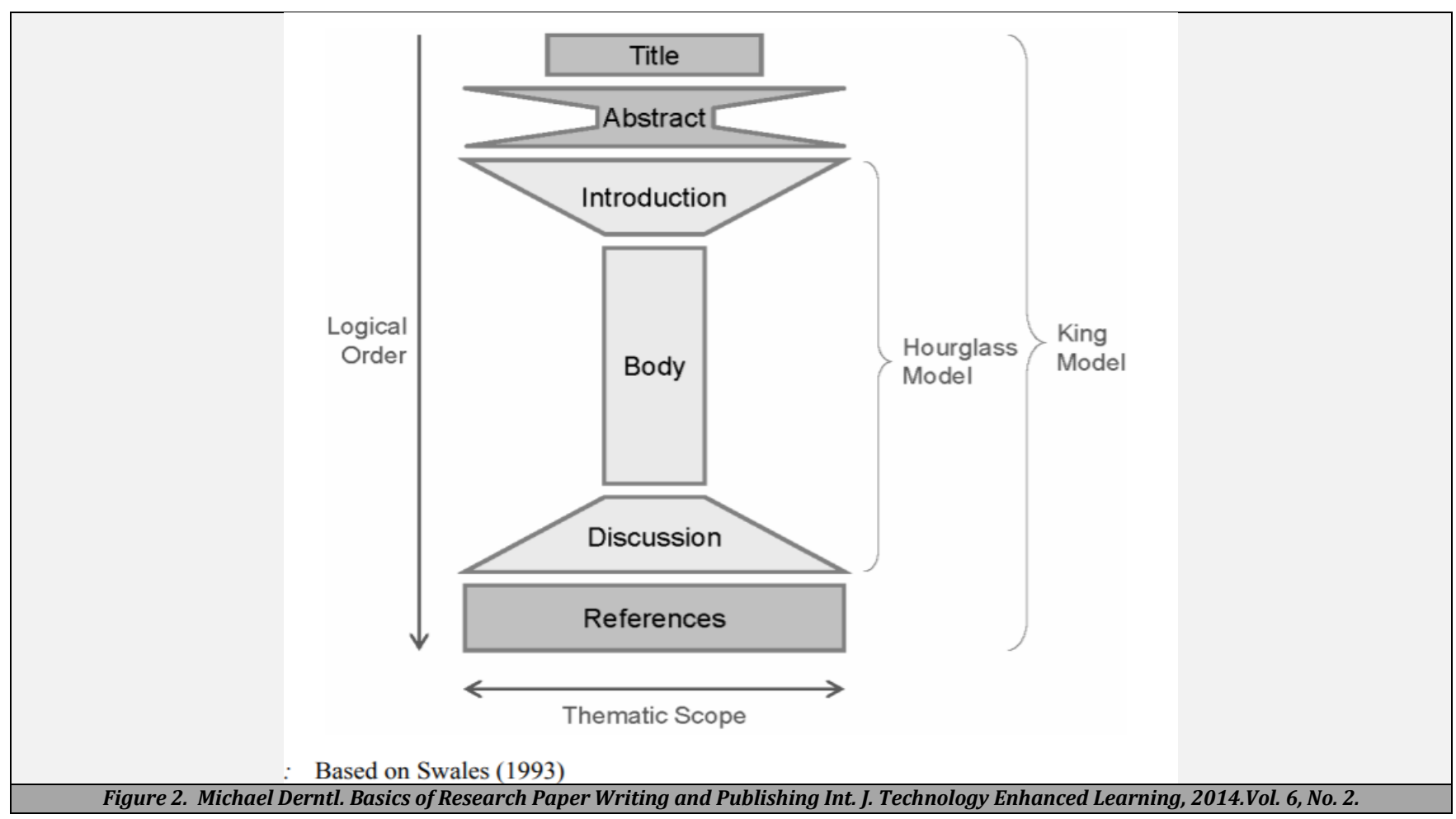

\section{CONCLUSIONS}

Conclusion should fall in line with the hypothesis of the research work. Conclusion and abstract are different. Conclusion is not copy from results and discussion. It is a brief summary of main or unique (novel) idea that emerges with penetrability and objectivity. The thought process may be started with a one liner conclusion by asking oneself what is this paper about? which can be expanded. It may be considered to be an extension of hypothesis and can have positive and negative aspects (limitations). The material method, tables and figures, statistical analysis, discussion and referencing are considered to be core scientific components.

Financial or other competing interests: None.

Disclosure forms provided by the authors are available with the full text of this article at jemds.com.

\section{REFERENCES}

[1] Derntl M. Basics of research paper writing and publishing. Int J Technology Enhanced Learning 2014;6(2):105-27.

[2] Meo SA. Anatomy and physiology of a scientific paper. Saudi J Biol Sci 2018;25(7):1278-83.

[3] Tullu MS. Writing the title and abstract for a research paper: being concise, precise and meticulous is the key. Saudi J Anaesth 2019;13(Suppl 1):S12-7.

[4] Thomas RM, Brubaker DL. Theses and dissertations: a guide to planning, research and writing. $2^{\text {nd }}$ edn. Corwin Press 2008.

[5] Liumbruno GM, Velati C, Pasqualetti P, et al. How to write a scientific manuscript for publication. Blood Transfus 2013;11(2):217-26.

[6] Mack CA. How to write a good scientific paper. Society of Photo-Optical Instrumentation Engineers (SPIE) 2018.

[7] Bajwa SJS, Sawhney C. Preparing manuscript: scientific writing for publication. Indian J Anaesth 2016;60(9):6748.

[8] Picardi N. Rules to be adopted for publishing a scientific paper. Ann Ital Chir 2016;87:1-3. 
[9] Erdemir F. How to write a materials and methods section of a scientific article? Turk J Urol 2013;39(Suppl 1):10-5.

[10] EQUATOR Network. Guidelines for Reporting Health Research. https://www.equator-network.org/reportingguidelines/

[11] Otzen T, Manterola C, Mora M, et al. Statements, recommendations, proposals, guidelines, checklists and scales available for reporting results in biomedical research and quality of conduct. A systematic review. Int J Morphol 2020;38(3):774-86.

[12] Fah TS, Aziz AFA. How to present research data? Malays Fam Physician 2006;1(2-3):82-5.

[13] Auvinen A. How do I write a scientific article--advice to a young researcher. Duodecim 2015;131(16):1460-6.
[14] Walsh K. Art of publication: the title, abstract and cover letter. Indian Dermatology Online Journal 2014;5(2):226.

[15] Cals JW, Kotz D. Effective writing and publishing scientific papers, part III: introduction. J Clin Epidemiol 2013;66(7):702.

[16] Bavdekar SB, Gogtay NJ. Writing an abstract for a research manuscript: providing an honest, succinct and complete summary. J Assoc Physicians India 2015;63(12):64-7.

[17] Gambescia SF. A brief on writing a successful abstract. Educ Health (Abingdon) 2013;26(2):122-5.

[18] Ali J. Manuscript rejection: causes and remedies. J Young Pharm 2010;2(1):3-6. 